[3] B. Nowak, On the Lipschitzian retraction of the unit ball in infinite-dimensional Banach spaces onto its boundary, Bull. Acad. Polon. Sci. 27 (11) (1979), 861-864.

[4] -, DJ-mappings and their homotopies (in Polish), Łódź 1980.

[5] R. D. Nussbau m, Degree theory for local condensing maps, J. M. A. Appl. 37 (1972), 741766.

[6] E. Zeidler, Vorlesungen ueber nichtlineare Funktionalanalysis, Teubner, Texte zur Mathematik, Leipzig 1976.

INSTITUTE OF MATHEMATICS AND PHYSICS, TECHNICAL UNIVERSITY RADOM, POLAND

Received April 20, 1982

\section{Connected subgroups of nuclear spaces}

by

\section{W. BANASZCZYK (Łódź) and J. GRABOWSKI (Warszawa)} Revised version June 14, 1982

Abstract. It is proved that closed and connected subgroups of nuclear spaces are real linear subspaces.

1. It is well known that a closed subgroup of a Lie group is a Lie subgroup. In the simplest linear case this amounts to the elementary fact that every closed and connected subgroup of $\boldsymbol{R}^{n}$ is its linear subspace. However, considering infinite-dimensional topological linear spaces we encounter essential differences. For example, the subset of all integer-valued functions in $L^{2}(0,1)$ is a closed and connected subgroup of $L^{2}(0,1)$ but it fails to contain any line.

Our aim in this note is to show that in the case of nuclear spaces the situation is analogous to the finite-dimensional case, namely:

THEOREM 1. Closed and connected subgroups of. nuclear spaces are real linear subspaces.

This theorem substanties a conjecture of W. Wojtyński and provides one more example that nuclear spaces are closer. to finite-dimensional spaces than normed spaces are.

After proving the theorem we have found that it can be derived also from the results of the first named author concerning unitary representations of groups which are quotiens of nuclear spaces by its closed subgroups.

We are indebted to many persons for stimulating discussions, especially to T. Dobrowolski, S. Kwapień, W. Wojtyński and T. Wolniewicz.

2. All linear spaces we shall deal in the sequel are assumed to be real. We shall obtain Theorem 1 as a consequence of the following

Theorem 2. Let $G$ be a subgroup of a real nuclear space $X$ such that for each neighbourhood $U$ of zero in $X \operatorname{span}(G \cap U)$ is dense in $X$. Then $G$ is dense in $X$.

In fact, assuming that Theorem 2 holds true let $G$ be a closed connected subgroup of a nuclear space $X$, let $X_{1}=\operatorname{cl}(\operatorname{span} G)$ and let $U$ be a neighbourhood of 0 in $X$.

The set $G \cap U$ generates $G$, hence $\operatorname{span}(G \cap U)$ is dense in $X_{1}$. Then, by Theorem 2, $G$ is dense in $X_{1}$, whence $G=\operatorname{cl} G=X_{1}$. 
Proof of Theorem 2. Since $X$ is a nuclear space, let $\left(\|\|_{i}, i \in I\right)$ be a family of seminorms defining the topology of $X$ such that:

$1^{\circ}$ For each $i \in I$ the seminorm \|\|$_{i}$ induces a Hilbert norm on the space $X / \operatorname{ker}\|\|_{i}$. The completion of $X / \operatorname{ker}\|\|_{i}$ with respect to this norm is a separable Hilbert space which will be denoted by $H_{i}$. We have the natural continuous linear operator $p_{i}: X \rightarrow H_{i}$.

$2^{\circ}$ For each $i \in I$ there is $k \in I$ such that \|\|$_{k} \geqslant\|\|_{i}$ and the canonical mapping $T_{i k}: H_{k} \rightarrow H_{i}$ is a Hilbert--Schmidt operator.

Let $x \in X, i \in I$ and $\varepsilon>0$. In order to prove our theorem we shall find $g \in G$ such that $\|g-x\|_{i}<\varepsilon$. Take $k \in I$ as in $2^{\circ}$ and $0<r<\varepsilon / 2\|\| T_{i k} \|$, where $\left\|T_{i k}\right\|$ is the Hilbert-Schmidt norm of the operator $T_{i k}$.

Since $K=\left\{g \in G:\|g\|_{k}<r\right\}$ is linearly dense in $X$, the set $p_{k}(K)$ is linearly dense in $H_{k}$. Hence there are $u_{1}, u_{2}, \ldots \in K$ such that the vectors $p_{k}\left(u_{1}\right), p_{k}\left(u_{2}\right), \ldots$ form a complete system of vectors in $H_{k}$, i.e. they are linearly independent and total in $H_{k}$.

Let $e_{1}, e_{2}, \ldots$ be the system obtained by the orthonormalization of the vectors $p_{k}\left(u_{1}\right), p_{k}\left(u_{2}\right), \ldots$ Then $p_{k}\left(u_{n}\right)=\sum_{m=1}^{n} \alpha_{n}^{m} e_{m}$, where obviously $\left|\alpha_{n}^{m}\right|$ $<r, n=1,2, \ldots, m=1, \ldots, n$

The vectors $p_{i}\left(u_{1}\right), p_{i}\left(u_{2}\right), \ldots$ are total in $H_{i}$ and $T_{i k} p_{k}\left(u_{n}\right)=p_{i}\left(u_{n}\right), n$ $=1,2, \ldots$ By omitting linearly dependent vectors in this sequence we obtain a complete system of vectors $p_{i}\left(u_{j_{1}}\right), p_{i}\left(u_{j_{2}}\right), \ldots$ in $H_{i}$. Let $f_{1}, f_{2}, \ldots$ be the orthonormalization of this system. We have then $p_{i}\left(u_{j_{n}}\right)=\sum_{m=1}^{n} \beta_{n}^{m} f_{m}, n$ $=1,2, \ldots$ As easily verified, the matrix of the operator $T_{i k}$ in the bases $e_{1}, e_{2}, \ldots$ and $f_{1}, f_{2}, \ldots$ has the form

$$
T_{i k}=\left[\begin{array}{cccc}
\gamma_{11} & \gamma_{12} & \gamma_{13} & \ldots \\
0 & \gamma_{22} & \gamma_{23} & \ldots \\
0 & 0 & \gamma_{33} & \ldots \\
\ldots & \ldots & \ldots & \ldots \\
\ldots & \ldots & \ldots & \ldots \\
\ldots & \ldots & \ldots & \ldots
\end{array}\right],
$$

where $\sum_{i, j=1}^{\infty}\left|\gamma_{i j}\right|^{2}=\left\|T_{i k} \mid\right\|^{2}$ and $\gamma_{n m}=0$ if $m<j(n)$. Moreover,

$$
\left|\beta_{n}^{n}\right|=\left|\gamma_{n j_{n}} \alpha_{j n}^{j n}\right|<r\left|\gamma_{n j_{n}}\right|, \quad n=1,2, \ldots
$$

Let $p_{i}(x)=\sum_{m=1}^{\infty} \delta_{m} f_{m}$. Take natural $l$ such that

$$
\sum_{m=l+1}^{\infty}\left|\delta_{m}\right|^{2}<3 \varepsilon^{2} / 4
$$

Now we can construct inductively a sequence $c_{l}, c_{l-1}, \ldots, c_{1}$ of integers so that

$$
p_{i}(x)-\sum_{m=1}^{l} c_{m} p_{i}\left(u_{j_{m}}\right)=\sum_{m=1}^{l} \varrho_{m} f_{m}+\sum_{m=l+1}^{\infty} \delta_{m} f_{m}
$$

where $\left|\varrho_{m}\right|<\left|\beta_{m}^{m}\right|<r\left|\gamma_{m, j_{m}}\right|, m=1, \ldots, l$.

Putting $g=\sum_{m=1}^{l} c_{m} u_{j_{m}}$ we have $g \in G$ and

$$
\|x-g\|_{i}^{2}=\sum_{m=1}^{l}\left|\varrho_{m}\right|^{2}+\sum_{m=l+1}^{\infty}\left|\delta_{m}\right|^{2}<r^{2} \sum_{m=1}^{l}\left|\gamma_{m j_{m}}\right|^{2}+3 \varepsilon^{2} / 4,
$$

whence

$$
\|x-g\|_{i}^{2}<r^{2}\left\|T_{i k}\right\| \|^{2}+3 \varepsilon^{2} / 4<\varepsilon^{2}, \quad \text { q.e.d. }
$$

3. The other well-known result in the theory of Lie groups is the theorem of Yamabe [2] which says that an arcwise connected subgroup of a Lie group is its Lie subgroup. Unlikely to the case of closed subgroups this theorem does not remain valid when we pass to infinite-dimensional nuclear spaces.

An easy example is the subgroup of $C^{\infty}(\boldsymbol{R})$, the nuclear space of infinitely differentiable functions on $\boldsymbol{R}$, consisting of those functions which take non-integer values only on subsets of finite measure.

This subgroup is arcwise-connected, contains constant integer-valued functions but does not contain the line of constant functions.

\section{References}

[1] A. Pietsch, Nuclear Locally Convex Spaces, Berlin 1972.

[2] H. Yamabe, On an arcwise connected subgroups of a Lie group, Osaka Math. J. 2 (1950), $13-14$.

INSTITUTI OF MATHFMATICS

tóIIÝ UNIVFRSITY, LÓDÝ, POLAND

and

INSTITUT: OF MATHEMATICS

UNIVIRSSTTY OF WARSAW, WARSZAWA, POLAND

Received June 14, 1982

Revised version October 12, 1982 